\title{
CONTINUED FRACTIONS OVER AN INNER PRODUCT SPACE
}

\author{
F. A. ROACH
}

1. Introduction. In this paper we introduce the notion of a continued fraction with elements which are points of a real inner product space and give three convergence theorems. Throughout this paper we assume that $S$ is a real inner product space and that $u$ is a particular point of $S$ with norm 1 . If $x$ is a point, the point $2((x, u)) u-x$ is referred to as the conjugate of $x$ with respect to $u$, and since we do not consider conjugation with respect to any other point, it will be denoted by $\bar{x}$. Notice that if $x$ and $y$ are points and $c$ is a real number, then

$$
\overline{x+y}=\bar{x}+\bar{y}, \quad \overline{c x}=\overline{c x}, \quad \overline{(\bar{x})}=x, \quad \text { and }\|\bar{x}\|=\|x\| .
$$

If $x$ is a point distinct from 0 , the symbol $1 / x$ will be used to denote the point $\bar{x} /\|x\|^{2}$. We assume there is adjoined to $S$ a "point at infinity" $\infty$ with the usual conventions; $1 / 0=\infty, 1 / \infty=0$, etc. Notice that if $T$ is a two dimensional subspace of $S$ which contains $u$, we may regard it as being the set of all complex numbers, with $u$ corresponding to unity, since within $T$, the transformation $1 / z$ is equivalent to the ordinary reciprocal transformation.

If, for $n=1,2,3, \cdots, b_{n}$ is a point, we refer to the expression

$$
\frac{1}{b_{1}+\frac{1}{b_{2}+\frac{1}{b_{3}+}} \text {. }}
$$

as a continued fraction over $S$. The symbol $f_{n}$ will be used to denote the $n$th approximate of (1.1). That is to say, $f_{1}=1 / b_{1}, f_{2}=1 /\left(b_{1}+1 / b_{2}\right)$, $f_{3}=1 /\left[b_{1}+1 /\left(b_{2}+1 / b_{3}\right)\right]$, etc. Of course, we say that $(1.1)$ converges if and only if $f_{1}, f_{2}, f_{3}, \cdots$ has a finite limit; when convergent, that limit is called the value of (1.1).

The following notation proves useful: for $n=1,2,3, \cdots$ and $k=1,2, \cdots, n$,

Received by the editors March 7, 1969. 


$$
\begin{aligned}
& a_{-1}^{n}=0, \quad a_{0}^{n}=1, \quad \text { and } \\
& a_{k}^{n}=b_{n-k+1}\left\|a_{k-1}^{n}\right\|^{2}+\bar{a}_{k-1}^{n}\left\|a_{k-2}^{n}\right\|^{2} .
\end{aligned}
$$

It follows by induction that for $n=1,2,3, \cdots$,

$$
f_{n}=\left(\left\|a_{n-1}^{n}\right\|^{2} Q_{n}^{n}\right) /\left\|a_{n}^{n}\right\|^{2} \text {. }
$$

2. A necessary condition for convergence. The following theorem, for the complex case-the case in which each partial denominator of (1.1) is a complex number-was given by von Koch [4]. For a proof and discussion of this result, see Wall [5, pp. 27-28].

THEOREM 1. If, for $n=1,2,3, \cdots, b_{n}$ is a point and the series $\sum\left\|b_{i}\right\|$ converges, then the continued fraction (1.1) diverges.

We will first establish an identity involving the symbols (1.2). Namely, that for $n=1,2,3, \cdots$

$$
\begin{aligned}
\left\|\left(\left\|a_{n-1}^{n}\right\|^{2}\left\|a_{n+1}^{n+1}\right\|^{2}\right) a_{n}^{n}-\left(\left\|a_{n}^{n+1}\right\|^{2}\left\|a_{n}^{n}\right\|^{2}\right) a_{n+1}^{n+1}\right\| \\
=\left(\prod_{i=1}^{n}\left\|a_{i}^{n}\right\|\right)\left(\prod_{i=1}^{n+1}\left\|a_{i}^{n+1}\right\|\right) .
\end{aligned}
$$

Making use of this identity and the convergence of the series named above, we will show that the approximants of (1.1) do not form a Cauchy sequence.

Since $S$ is an inner product space, the square of the left-hand side of (2.1) may be written as

$\left\|Q_{n+1}^{n+1}\right\|^{2}\left\|Q_{n}^{n}\right\|^{2}\left[\left\|Q_{n-1}^{n}\right\|^{4}\left\|Q_{n+1}^{n+1}\right\|^{2}-2\left\|Q_{n}^{n+1}\right\|^{2}\left(\left(Q_{n}^{n}, Q_{n+1}^{n+1}\right)\right)+\left\|Q_{n}^{n+1}\right\|\left\|^{4}\right\| Q_{n}^{n} \|^{2}\right]$.

With this, we see that the left-hand side of (2.1) is

$$
\left\|a_{n+1}^{n+1}\right\| \cdot\left\|a_{n}^{n}\right\| \cdot\|\| a_{n-1}^{n}\left\|^{2} Q_{n+1}^{n+1}-\right\| a_{n}^{n+1}\left\|^{2} Q_{n}^{n}\right\|,
$$

which, with the aid of (1.2), may be written as

(2.2) $\left\|a_{n+1}^{n+1}\right\| \cdot\left\|a_{n}^{n}\right\| \cdot\|\| a_{n-2}^{n}\left\|^{2}\right\| a_{n}^{n+1}\left\|^{2} Q_{n-1}^{n}-\right\| a_{n-1}^{n+1}\left\|^{2}\right\| a_{n-1}^{n}\left\|^{2} a_{n}^{n+1}\right\|$.

It is now easily seen that (2.1) holds true in case $n=1$. The truth of (2.1) follows at once by induction.

Dividing both sides of (2.1) by $\left\|Q_{n+1}^{n+1}\right\|\left\|^{2}\right\| Q^{n} \|^{2}$, we obtain

$$
\left\|f_{n}-f_{n+1}\right\|=\left(\prod_{i=1}^{n-1}\left\|a_{i}^{n-1}\right\|\right)\left(\prod_{i=1}^{n}\left\|a_{i}^{n}\right\|\right) /\left(\left\|a_{n+1}^{n+1}\right\|\left\|Q_{n}^{n}\right\|\right) .
$$

We now introduce some additional notation: for $n=1,2,3, \cdots$, 


$$
D_{n}=\left\|b_{n}\right\|\left\|b_{n-1}+1 / b_{n}\right\| \cdots\left\|b_{1}+\underline{1} / \overline{b_{2}}+\cdots+\underline{1} / \overline{b_{n}}\right\| .
$$

Since

$$
1 / \sqrt{b_{n-k}}+1 / \sqrt{b_{n-k+1}}+\cdots+\underline{1} / \overline{b_{n}}=\left(\left\|Q_{k}^{n}\right\|^{2} Q_{k+1}^{n}\right) /\left\|a_{k+1}^{n}\right\|,
$$

by means of a simple calculation, the right-hand side of (2.3) may be reduced to $1 /\left(D_{n} D_{n+1}\right)$. Thus we have

$$
\left\|f_{n}-f_{n+1}\right\|=1 /\left(D_{n} D_{n+1}\right) \text {. }
$$

Clearly,

$$
\begin{aligned}
& D_{1} \leqq 1+\left\|b_{1}\right\|, \\
& D_{2} \leqq\left\|\left(\left\|b_{2}\right\| b_{1}+b_{2} /\left\|b_{2}\right\|\right)\right\| \leqq\left(1+\left\|b_{1}\right\|\right)\left(1+\left\|b_{2}\right\|\right),
\end{aligned}
$$

and, by induction, it follows that

$$
D_{n} \leqq\left(1+\left\|b_{1}\right\|\right)\left(1+\left\|b_{2}\right\|\right) \cdots\left(1+\left\|b_{n}\right\|\right) .
$$

However, if the series $\sum\left\|b_{i}\right\|$ is convergent, then the product $\Pi\left(1+\left\|b_{i}\right\|\right)$ is also convergent. Therefore, in view of (2.6), the sequence $D_{1}, D_{2}, D_{3}, \cdots$ does not increase without bound and so, with the aid of (2.5), we see that $f_{1}, f_{2}, f_{3}, \cdots$ does not form a Cauchy sequence. From this we conclude that the divergence of the series $\sum\left\|b_{i}\right\|$ is necessary for the convergence of the continued fraction $(1.1)$.

REMARK 2.1. Notice that in the proof of the above theorem, we did not need to use directly the fact that $\bar{x}=2((x, u)) u-x$. In fact, if $T$ is a linear transformation from $S$ onto $S$ of norm 1 such that $T^{2}$ is the identity transformation, we may interpret $\bar{x}$ to be $T(x)$ and this theorem remains true.

REMARK 2.2. One might raise the question as to whether the above theorem can be generalized in a manner similar to the generalization of von Koch's theorem due to Scott and Wall [1].

3. A sufficient condition for convergence. The following theorem, for the complex case, may be found in a paper by Thron [2]. In this case, if $c=0$, the theorem reduces to a theorem due to Worpitzky [6]. For a discussion of this result, see Wall [5, pp. 42-44]. We use the term convergence set to mean a point set having the property that if $b_{n}$ lies in it, $n=1,2,3, \cdots$, the continued fraction (1.1) converges. If $M$ is a point set by the value region associated with $M$ is meant the set of all points which, for some sequence $b_{1}, b_{2}, b_{3}, \cdots$ from $M$, are either one of the approximants of (1.1) or else, in case (1.1) converges, the value of (1.1). 
TheOREM 2. Suppose that $S$ is complete and $c$ is a real number. If $M$ is the set of all points $z$ such that

$$
\|z-c u\| \geqq\left(c^{2}+4\right)^{1 / 2},
$$

then $M$ is a convergence set. Moreover, the value region associated with $M$ is the set of all points $z$ such that

$$
\|2 z+c u\| \leqq\left(c^{2}+4\right)^{1 / 2} .
$$

We will first establish that the value region is given by (3.2). To this end, let $M_{1}$ denote $M$ and $V_{1}$ denote $1 / M_{1}$ (i.e., $V_{1}$ is the set of all points $z$ such that, for some point $x$ of $\left.M_{1}, z=1 / x\right)$. Let $M_{2}$ denote the point set $M_{1}+V_{1}$-the set of all points $z$ such that for some point $x$ of $M_{1}$ and some point $y$ of $V_{1}, z=x+y$-and let $V_{2}$ denote $1 / M_{2}$. Denote by $M_{3}$ the point set $M_{1}+V_{2}$ and by $V_{3}$, the point set $1 / M_{3}$, etc. Thus, for $n=1,2,3, \cdots, M_{n+1}$ is $M_{1}+V_{n}$ and $V_{n+1}$ is $1 / M_{n+1}$. By a simple argument, we see that $V_{1}$ is the set of all points $z$ such that

$$
\|z-(1 / a+1 / b) u / 2\| \leqq(1 / a-1 / b) / 2
$$

where $a=c+\left(c^{2}+4\right)^{1 / 2}$ and $b=c-\left(c^{2}+4\right)^{1 / 2}$. We also find that $V_{2}$ is the set of all points $z$ such that

$$
\begin{aligned}
\|z-[1 /(a+1 / b)+1 /(b+1 / a)] u / 2\| & \\
& \leqq[1 /(a+1 / b)-1 /(b+1 / a)] / 2,
\end{aligned}
$$

etc. Since

$$
1 / \bar{a}+\underline{1} / \bar{b}+\underline{1} / \bar{a}+\cdots
$$

has value $-\left[c-\sqrt{ }\left(c^{2}+4\right)\right]$ and

$$
\underline{1} / \bar{b}+\underline{1} / \bar{a}+\underline{1} / \bar{b}+\cdots
$$

value $-\left[c+\sqrt{ }\left(c^{2}+4\right)\right]$, we see that $V$ is the set of all points $z$ such that (3.2) holds true.

Suppose that, for $n=1,2,3, \cdots, b_{n}$ lies in $M$ and let $K_{n}$ denote the point set

$$
1 / \bar{b}_{1}+\underline{1} / \bar{b}_{2}+\cdots+\underline{1} / \overline{b_{n}+V} .
$$

Now, for $n=1,2,3, \cdots$, let $b_{n}^{\prime}$ denote the complex number $x$ such that $|x|=\left\|b_{n}\right\|, \operatorname{Re}(x)=\left(\left(b_{n}, u\right)\right)$, and $\operatorname{Im}(x) \geqq 0$. Denote by $M^{\prime}$ the set of all complex numbers $z$ such that, with $u=1$, (3.1) holds true and by $V^{\prime}$ the corresponding value region. Also, for $n=1,2,3, \cdots$, let $K_{n}^{\prime}$ denote the set 


$$
1 / \overline{b_{1}^{\prime}}+\underline{1} / \overline{b_{2}^{\prime}}+\cdots+\underline{1} / \overline{b_{n}^{\prime}+V^{\prime}} .
$$

It is not difficult to see that $K_{n}$ is a sphere together with its interior, $K_{n}^{\prime}$ is a circle together with its interior, and that the radius of $K_{n}$ is the same as the radius of $K_{n}^{\prime}$. It is also easily seen that $K_{n+1}$ is a subset of $K_{n}$ and that $K_{n+1}^{\prime}$ is a subset of $K_{n}^{\prime}$.

We will now show that the radius of $K_{n}^{\prime}$ tends to 0 as $n$ increases. We have from [2] that

$$
\underline{1} \overline{b_{1}^{\prime}}+\underline{1} \overline{b_{2}^{\prime}}+\underline{1} \overline{b_{3}^{\prime}}+\cdots
$$

converges. Let $v$ denote the value of this continued fraction and suppose that $v^{\prime}$ is a complex number which belongs to each one of $K_{1}^{\prime}$, $K_{2}^{\prime}, K_{3}^{\prime}, \cdots$ (Clearly $v$ belongs to each of these sets.) Thus, we see that there exists a sequence $x_{1}, x_{2}, x_{3}, \cdots$ such that, for $n=1,2$, $3, \cdots, x_{n}$ lies in $V^{\prime}$ and

$$
v^{\prime}=\underline{1} / \overline{b_{1}^{\prime}}+\underline{1} \overline{b_{2}^{\prime}}+\cdots+\underline{1} / \overline{b_{n}^{\prime}+x_{n}} .
$$

There exists a complex number $x$ and a subsequence $s$ of $x_{1}, x_{2}$, $x_{3}, \cdots$ such that $s$ converges to $x$. For $n=1,2,3, \cdots$, let

$$
v_{n}=\underline{1} / \overline{b_{1}^{\prime}}+\underline{1} / \overline{b_{2}^{\prime}}+\cdots+\underline{1} / \overline{b_{n}^{\prime}+x} .
$$

Making use of the well-known recursion formulas for computing the value of $v_{n}$, we see that $v_{1}, v_{2}, v_{3}, \cdots$ has limit $v$. Notice that for $n=1,2,3, \cdots$, the continuous transformation

$$
1 / \overline{b_{1}^{\prime}}+\underline{1} \overline{b_{2}^{\prime}}+\cdots+\underline{1} \overline{b_{n}^{\prime}+z},
$$

maps $V^{\prime}$ onto a subset of $V^{\prime}$. It is not difficult to see that the subsequence of $v_{1}, v_{2}, v_{3}, \ldots$ which corresponds to $s$ must converge to $v^{\prime}$. Hence, $v=v^{\prime}$ and from this we conclude that the radius of $K_{n}$ tends to 0 as $n$ increases. Therefore, if each one of $b_{1}, b_{2}, b_{3}, \cdots$ lies in $M$, the continued fraction (1.1) converges.

REMARK 3.1. In view of the proof given above, one might suppose that all questions of convergence of continued fractions over $S$ could be reduced to questions of convergence of ordinary continued fractions in this manner. However, this is not the case. Consider the following example in which $S=E_{3}$ and $u=(1,0,0)$. For $n=1,2$, $3, \cdots$, let $b_{2 n-1}$ denote $(0,1,0)$ and $b_{2 n}$ denote $(0,0,1)$. In this case, both $b_{2 n-1}^{\prime}$ and $b_{2 n}^{\prime}$ (as used above) would be the complex number $i$ and the continued fraction 


$$
\underline{1} \bar{i}+\underline{1} / \bar{i}+\underline{1} / \bar{i}+\cdots
$$

is divergent. But with these values for $b_{1}, b_{2}, b_{3}, \cdots,(1.1)$ is convergent. This may be seen by observing that if, for $n=1,2,3, \cdots$, $\left(x_{n}, y_{n}, z_{n}\right)$ denotes the $n$th approximant of $(1.1)$, then $x_{n}=0$ and the complex number $y_{n}+i z_{n}$ is the $n$th approximant of the periodic continued fraction

$$
\underline{1} \overline{-1}+\underline{1} \bar{i}+\underline{1} \overline{-1}+\cdots
$$

which is convergent. (See Wall [5, p. 37].)

REMARK 3.2. The region $M$ of Theorem 2 is "best" in the sense that if $r$ is a positive number which is less than $\left(c^{2}+4\right)^{1 / 2}$ and the righthand side of (3.1) is replaced by $r$, the resulting statement does not hold true. This may be seen by simply considering the real case.

4. Van Vleck's theorem. The theorem of this section, for the complex case, was first given by Van Vleck [3]. A proof differing from Van Vleck's and some extensions of this theorem due to Scott and Wall [1] are given by Wall [5, pp. 123-134].

TheOREM 3. Suppose that $S$ is complete, $c$ is a positive number, and let $M$ denote the set of all points $z$ such that

$$
\|z-((z, u)) u\| \leqq c((z, u)) .
$$

If $b_{1}$ is a point such that $\left(\left(b_{1}, u\right)\right)>0$ and, for $n=1,2,3, \cdots, b_{n}$ lies in $M$, then the continued fraction (1.1) converges if and only if the series $\sum\left\|b_{i}\right\|$ diverges.

If the series $\sum\left\|b_{i}\right\|$ converges, we have by Theorem 1 that the continued fraction (1.1) diverges. Hence, suppose that this series is divergent. Notice that since, for $n=1,2,3, \cdots, b_{n}$ belongs to the "cone" $M$, the series $\sum\left(\left(b_{i}, u\right)\right)$ diverges also. This, together with the fact that $\left(\left(b_{1}, u\right)\right)>0$, implies that the continued fraction

$$
\underline{1} / \overline{\left(\left(b_{1}, u\right)\right)}+\underline{1} / \overline{\left(\left(b_{2}, u\right)\right)}+\underline{1} / \overline{\left(\left(b_{3}, u\right)\right)}+\cdots
$$

converges since $\left(\left(b_{n}, u\right)\right) \geqq 0, n=2,3,4, \cdots$. We will use this fact to establish that the approximants of (1.1) form a Cauchy sequence.

The point set $M$ has the property that if $z$ and $z^{\prime}$ belong to it, then so do $z+z^{\prime}, \bar{z}$, and any nonnegative multiple of $z$. Therefore, if $m$ and $n$ are positive integers and $m>n$,

$$
1 / \overline{b_{n}}+\underline{1} / \overline{b_{n+1}}+\cdots+\underline{1} / \overline{b_{m}}
$$

lies in $M$. Now, for $n=1,2,3, \cdots$, let $K_{n}$ denote the point set 


$$
1 / \overline{b_{1}}+\underline{1} / \overline{b_{2}}+\cdots+\underline{1} \overline{b_{n}+M} .
$$

Notice that for $m>n, f_{m}$ lies in $K_{n}$. As in the proof of Theorem 2, we will show that $d_{n}$, the diameter of $K_{n}$ tends to zero as $n$ increases. Clearly, $d_{1} \leqq 1 /\left(\left(b_{1}, u\right)\right)$ since $\left\|b_{1}\right\| \geqq\left(\left(b_{1}, u\right)\right)$. We also have, by means of a geometrical argument, that the point set $1 /\left(b_{2}+M\right)$ is a subset of the spherical ball with center $u / 2\left(\left(b_{2}, u\right)\right)$ and radius $1 / 2\left(\left(b_{2}, u\right)\right)$. From this it follows that

$$
d_{2} \leqq\left|1 /\left[\left(\left(b_{1}, u\right)\right)+1 /\left(\left(b_{2}, u\right)\right)\right]-1 /\left(\left(b_{1}, u\right)\right)\right| .
$$

By a simple argument we conclude that, for $n=2,3,4, \cdots, d_{n}$ does not exceed the absolute value of the difference between the $n$th and the $(n+1)$ st approximants of (4.1). However, (4.1) converges and hence we see that $f_{1}, f_{2}, f_{3}, \cdots$ is a Cauchy sequence.

\section{REFERENCES}

1. W. T. Scott and H. S. Wall, On the convergence and divergence of continued fractions, Amer. J. Math. 69 (1947), 551-561. MR 9, 28.

2. W. J. Thron, Twin convergence regions for continued fractions $b_{0}+K\left(1 / b_{n}\right)$. II, Amer. J. Math. 71 (1949), 112-120. MR 10, 292.

3. E. V. Van Vleck, On the convergence of continued fractions with complex elements, Trans. Amer. Math. Soc. 2 (1901), 215-233.

4. H. von Koch, Sur un théorème de Steltjes et sur les fractions continues, Bull. Soc. Math. France 23 (1895), 23-40.

5. H. S. Wall, Analytic theory of continued fractions, Van Nostrand, Princeton, N. J., 1948. MR 10, 32.

6. J. Worpitzky, Untersuchungen über die Entwickelung der monodronen und monogenen Funktionen durch Kettenbrüche, Friedrichs-Gymnasium und Realschule, Jahresbericht, Berlin, 1865, pp. 3-39.

UNIVERSITY OF GEORGIA 